\title{
Alternative Approaches for Generating Bodies of Grammar Rules
}

\author{
Gabriel Infante-Lopez and Maarten de Rijke \\ Informatics Institute, University of Amsterdam \\ \{infante,mdr\}@science.uva.nl
}

\begin{abstract}
We compare two approaches for describing and generating bodies of rules used for natural language parsing. In today's parsers rule bodies do not exist a priori but are generated on the fly, usually with methods based on $n$-grams, which are one particular way of inducing probabilistic regular languages. We compare two approaches for inducing such languages. One is based on $n$-grams, the other on minimization of the Kullback-Leibler divergence. The inferred regular languages are used for generating bodies of rules inside a parsing procedure. We compare the two approaches along two dimensions: the quality of the probabilistic regular language they produce, and the performance of the parser they were used to build. The second approach outperforms the first one along both dimensions.
\end{abstract}

\section{Introduction}

$N$-grams have had a big impact on the state of the art in natural language parsing. They are central to many parsing models (Charniak, 1997; Collins, 1997, 2000; Eisner, 1996), and despite their simplicity $n$-gram models have been very successful. Modeling with $n$-grams is an induction task (Gold, 1967). Given a sample set of strings, the task is to guess the grammar that produced that sample. Usually, the grammar is not be chosen from an arbitrary set of possible grammars, but from a given class. Hence, grammar induction consists of two parts: choosing the class of languages amongst which to search and designing the procedure for performing the search. By using $n$-grams for grammar induction one addresses the two parts in one go. In particular, the use of $n$-grams implies that the solution will be searched for in the class of probabilistic regular languages, since $n$-grams induce probabilistic automata and, consequently, probabilistic regular languages. However, the class of probabilistic regular languages induced using $n$-grams is a proper subclass of the class of all probabilistic regular languages; $n$-grams are incapable of capturing long-distance relations between words. At the technical level the restricted nature of $n$-grams is witnessed by the special structure of the automata induced from them, as we will see in Section 4.2.
$N$-grams are not the only way to induce regular languages, and not the most powerful way to do so. There is a variety of general methods capable of inducing all regular languages (Denis, 2001; Carrasco and Oncina, 1994; Thollard et al., 2000). What is their relevance for natural language parsing? Recall that regular languages are used for describing the bodies of rules in a grammar. Consequently, the quality and expressive power of the resulting grammar is tied to the quality and expressive power of the regular languages used to describe them. And the quality and expressive power of the latter, in turn, are influenced directly by the method used to induce them. These observations give rise to a natural question: can we gain anything in parsing from using general methods for inducing regular languages instead of methods based on $n$-grams? Specifically, can we describe the bodies of grammatical rules more accurately and more concisely by using general methods for inducing regular languages?

In the context of natural language parsing we present an empirical comparison between algorithms for inducing regular languages using $n$ grams on the one hand, and more general algorithms for learning the general class of regular language on the other hand. We proceed as follows. We generate our training data from the Wall Street Journal Section of the Penn Tree Bank (PTB), by transforming it to projective dependency structures, following (Collins, 1996), and extracting rules from the result. These rules are used as training material for the rule induction algorithms we consider. The automata produced this way are then used to build grammars which, in turn, are used for parsing.

We are interested in two different aspects of the use of probabilistic regular languages for natural language parsing: the quality of the induced automata and the performance of the resulting parsers. For evaluation purposes, we use two different metrics: perplexity for the first aspect and percentage of correct attachments for the second. The main results of the paper are that, measured in terms of perplexity, the automata induced by algorithms other than $n$-grams describe the rule bodies better than automata induced using $n$-gram-based algorithms, and that, moreover, the gain in automata quality 
is reflected by an improvement in parsing performance. We also find that the parsing performance of both methods ( $n$-grams vs. general automata) can be substantially improved by splitting the training material into POS categories. As a side product, we find empirical evidence to suggest that the effectiveness of rule lexicalization techniques (Collins, 1997; Sima'an, 2000) and parent annotation techniques (Klein and Manning, 2003) is due to the fact that both lead to a reduction in perplexity in the automata induced from training corpora.

Section 2 surveys our experiments, and later sections provide details of the various aspects. Section 3 offers details on our grammatical framework, PCW-grammars, on transforming automata to PCW-grammars, and on parsing with PCWgrammars. Section 4 explains the starting point of this process: learning automata, and Section 5 reports on parsing experiments. We discuss related work in Section 6 and conclude in Section 7.

\section{Overview}

We want to build grammars using different algorithms for inducing their rules. Our main question is aimed at understanding how different algorithms for inducing regular languages impact the parsing performance with those grammars. A second issue that we want to explore is how the grammars perform when the quality of the training material is improved, that is, when the training material is separated into part of speech (POS) categories before the regular language learning algorithms are run.

We first transform the PTB into projective dependencies structures following (Collins, 1996). From the resulting tree bank we delete all lexical information except POS tags. Every POS in a tree belonging to the tree-bank has associated to it two different, possibly empty, sequences of right and left dependents, respectively. We extract all these sequences for all trees, producing two different sets containing right and left sequences of dependents respectively.

These two sets form the training material used for building four different grammars. The four grammars differ along two dimensions: the number of automata used for building them and the algorithm used for inducing the automata. As to the latter dimension, in Section 4 we use two algorithms: the Minimum Discriminative Information (MDI) algorithm, and a bigram-based algorithm. As to the former dimension, two of the grammars are built using only two different automata, each of which is built using the two sample set generated from the PTB. The other two grammars were built using two automata per POS, exploiting a split of the train- ing samples into multiple samples, two samples per POS, to be precise, each containing only those samples where the POS appeared as the head.

The grammars built from the induced automata are so-called PCW-grammars (see Section 3), a formalism based on probabilistic context free grammars (PCFGs); as we will see in Section 3, inferring them from automata is almost immediate.

\section{Grammatical Framework}

We briefly detail the grammars we work with (PCW-grammars), how automata give rise to these grammars, and how we parse using them.

\subsection{PCW-Grammars}

We need a grammatical framework that models rule bodies as instances of a regular language and that allows us to transform automata to grammars as directly as possible. We decided to embed them in the general grammatical framework of CW-grammars (Infante-Lopez and de Rijke, 2003): based on PCFGs, they have a clear and wellunderstood mathematical background and we do not need to implement ad-hoc parsing algorithms.

A probabilistic constrained W-grammar (PCWgrammar) consists of two different sets of PCF-like rules called pseudo-rules and meta-rules respectively and three pairwise disjoint sets of symbols: variables, non-terminals and terminals. Pseudorules and meta-rules provide mechanisms for building 'real' rewrite rules. We use $\alpha \stackrel{w}{\Longrightarrow} \beta$ to indicate that $\alpha$ should be rewritten as $\beta$. In the case of PCWgrammars, rewrite rules are built by first selecting a pseudo-rule, and then using meta-rules for instantiating all the variables in the body of the pseudo-rule.

To illustrate these concepts, we provide an example. Let $W=(V, N T, T, S, \stackrel{m}{\longrightarrow}, \stackrel{s}{\longrightarrow})$ be a CWgrammar such that the set of variable, non-terminals

\begin{tabular}{l|l}
\multicolumn{1}{c|}{ meta-rules } & \multicolumn{1}{|c}{ pseudo-rules } \\
\hline$\overline{A d j} \stackrel{m}{\longrightarrow} 0.5 \overline{A d j} A d j$ & $S \stackrel{s}{\longrightarrow} \overline{A d j}$ Noun \\
$\overline{A d j} \stackrel{m}{\longrightarrow}_{0.5} A d j$ & Adj $\stackrel{s}{\longrightarrow}_{0.1}$ big \\
& Noun $\stackrel{\mathrm{S}}{\longrightarrow}$ ball $^{\longrightarrow}$ \\
& $\vdots$
\end{tabular}

and terminals are defined as follows: $V=\{\overline{A d j}\}$, $N T=\{S$, Adj, Noun $\}, T=\{$ ball, big, fat, red, green, ... $\}$. As usual, the numbers attached to the arrows indicate the probabilities of the rules. The rules defined by $W$ have the following shape: $\bar{S} \stackrel{w}{\Longrightarrow} A d j^{*}$ Noun. Suppose now that we want to build the rule $\bar{S} \stackrel{w}{\Longrightarrow} A d j$ Adj Noun. We take the pseudo-rule $\bar{S} \stackrel{s}{\longrightarrow} \overline{A d j}$ Noun and instantiate the 
variable $\overline{A d j}$ with $A d j A d j$ to get the desired rule. The probability for it is $1 \times 0.5 \times 0.5$, that is, the probability of the derivation for Adj Adj times the probability of the pseudo-rule used. Trees for this particular grammar are flat, with a main node $S$ and all the adjectives in it as daughters. An example derivation is given in Figure 1(a).

\subsection{From Automata to Grammars}

Now that we have introduced PCW-grammars, we describe how we build them from the automata that we are going to induce in Section 4. Since we will induce two families of automata ("ManyAutomata" where we use two automata per POS, and "One-Automaton" where we use only two automata to fit every POS), we need to describe two automata-to-grammar transformations.

Let's start with the case where we build two automata per POS. Let $w$ be a POS in the PTB; let $A_{L}^{w}$ and $A_{R}^{w}$ be the two automata associated to it. Let $G_{L}^{w}$ and $G_{R}^{w}$ be the PCFGs equivalent to $A_{L}^{w}$ and $A_{R}^{w}$, respectively, following (Abney et al., 1999), and let $S_{L}^{w}$ and $S_{R}^{w}$ be the starting symbols of $G_{L}^{w}$ and $G_{R}^{w}$, respectively. We build our final grammar $G$ with starting symbol $S$, by defining its meta-rules as the disjoint union of all rules in $G_{L}^{w}$ and $G_{R}^{w}$ (for all POS $w$ ), its set of pseudo-rules as the union of the sets $\left\{W \stackrel{s}{\longrightarrow} S_{L}^{w} w S_{R}^{w}\right.$ and $\left.S \stackrel{s}{\longrightarrow} 1 S_{L}^{w} w S_{R}^{w}\right\}$, where $W$ is a unique new variable symbol associated to $w$.

When we use two automata for all parts of speech, the grammar is defined as follows. Let $A_{L}$ and $A_{R}$ be the two automata learned. Let $G_{L}$ and $G_{R}$ be the PCFGs equivalent to $A_{L}$ and $A_{R}$, and let $S_{L}$ and $S_{R}$ be the starting symbols of $G_{L}$ and $G_{R}$, respectively. Fix a POS $w$ in the PTB. Since the automata are deterministic, there exist states $S_{L}^{w}$ and $S_{R}^{w}$ that are reachable from $S_{L}$ and $S_{R}$, respectively, by following the arc labeled with $w$. Define a grammar as in the previous case. Its starting symbol is $S$, its set of meta-rules is the disjoint union of all rules in $G_{L}^{w}$ and $G_{R}^{w}$ (for all POS $w$ ), its set of pseudorules is $\left\{W \stackrel{s}{\longrightarrow} 1 S_{L}^{w} w S_{R}^{w}, S \stackrel{s}{\longrightarrow} S_{L}^{w} w S_{R}^{w}\right.$ : $w$ is a POS in the PTB and $W$ is a unique new variable symbol associated to $w$ \}.

\subsection{Parsing PCW-Grammars}

Parsing PCW-grammars requires two steps: a generation-rule step followed by a tree-building step. We now explain how these two steps can be carried out in one go. Parsing with PCW-grammars can be viewed as parsing with PCF grammars. The main difference is that in PCW-parsing derivations for variables remain hidden in the final tree. To clarify this, consider the trees depicted in Figure 1; the tree in part (a) is the CW-tree corresponding to the word red big green ball, and the tree in part (b) is the same tree but now the instantiations of the metarules that were used have been made visible.
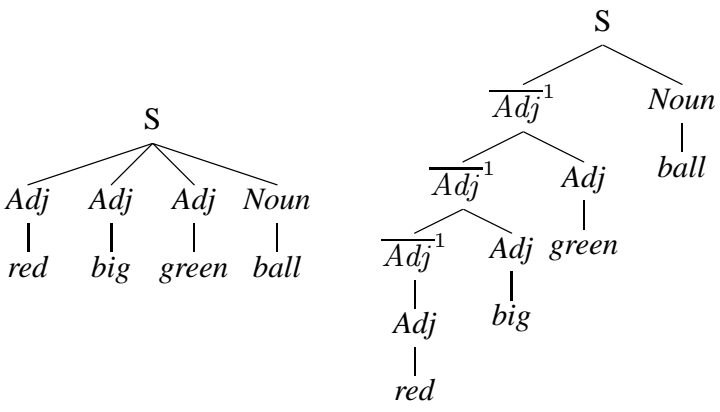

(a)

(b)

Figure 1: (a) A tree generated by $W$. (b) The same tree with meta-rule derivations made visible.

To adapt a PCFG to parse CW-grammars, we need to define a PCF grammar for a given PCWgrammar by adding the two sets of rules while making sure that all meta-rules have been marked somehow. In Figure 1(b) the head symbols of meta-rules have been marked with the superscript 1 . After parsing the sentence with the PCF parser, all marked rules should be collapsed as shown in part (a).

\section{Building Automata}

The four grammars we intend to induce are completely defined once the underlying automata have been built. We now explain how we build those automata from the training material. We start by detailing how the material is generated.

\subsection{Building the Sample Sets}

We transform the PTB, sections 2-22, to dependency structures, as suggested by (Collins, 1999). All sentences containing CC tags are filtered out, following (Eisner, 1996). We also eliminate all word information, leaving only POS tags. For each resulting dependency tree we extract a sample set of right and left sequences of dependents as shown in Figure 2. From the tree we generate a sample set with all right sequences of dependents $\{\epsilon, \epsilon, \epsilon\}$, and another with all left sequences $\{\epsilon, \epsilon$, red big green $\}$. The sample set used for automata induction is the union of all individual tree sample sets.

\subsection{Learning Probabilistic Automata}

Probabilistic deterministic finite state automata (PDFA) inference is the problem of inducing a stochastic regular grammar from a sample set of strings belonging to an unknown regular language. The most direct approach for solving the task is by 


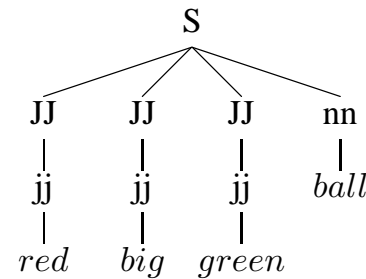

(a)

\begin{tabular}{cc|cc|cc}
\hline \multicolumn{2}{c}{$j j$} & \multicolumn{2}{c}{$j j$} & \multicolumn{2}{c}{$n n$} \\
\hline left & right & left & right & left & right \\
\hline$\epsilon$ & $\epsilon$ & $\epsilon$ & $\epsilon$ & red big green & $\epsilon$ \\
\hline \multicolumn{4}{c}{ (c) }
\end{tabular}

(c)

Figure 2: (a), (b) Dependency representations of Figure 1. (c) Sample instances extracted from this tree.

using $n$-grams. The $n$-gram induction algorithm adds a state to the resulting automaton for each sequence of symbols of length $n$ it has seen in the training material; it also adds an arc between states $a \beta$ and $\beta b$ labeled $b$, if the sequence $a \beta b$ appears in the training set. The probability assigned to the $\operatorname{arc}(a \beta, \beta b)$ is proportional to the number of times the sequence $a \beta b$ appears in the training set. For the remainder, we take $n$-grams to be bigrams.

There are other approaches to inducing regular grammars besides ones based on $n$-grams. The first algorithm to learn PDFAs was ALERGIA (Carrasco and Oncina, 1994); it learns cyclic automata with the so-called state-merging method. The Minimum Discrimination Information (MDI) algorithm (Thollard et al., 2000) improves over ALERGIA and uses Kullback-Leibler divergence for deciding when to merge states. We opted for the MDI algorithm as an alternative to $n$-gram based induction algorithms, mainly because their working principles are radically different from the $n$-gram-based algorithm. The MDI algorithm first builds an automaton that only accepts the strings in the sample set by merging common prefixes, thus producing a tree-shaped automaton in which each transition has a probability proportional to the number of times it is used while generating the positive sample.

The MDI algorithm traverses the lattice of all possible partitions for this general automaton, attempting to merge states that satisfy a trade-off that can be specified by the user. Specifically, assume that $A_{1}$ is a temporary solution of the algorithm and that $A_{2}$ is a tentative new solution derived from $A_{1} . \Delta\left(A_{1}, A_{2}\right)=D\left(A_{0} \| A_{2}\right)-D\left(A_{0} \| A_{1}\right)$ denotes the divergence increment while going from $A_{1}$ to $A_{2}$, where $D\left(A_{0} \| A_{i}\right)$ is the Kullback-Leibler divergence or relative entropy between the two distributions generated by the corresponding au- tomata (Cover and Thomas, 1991). The new solution $A_{2}$ is compatible with the training data if the divergence increment relative to the size reduction, that is, the reduction of the number of states, is small enough. Formally, let a lpha denote a compatibility threshold; then the compatibility is satisfied if $\frac{\Delta\left(A_{1}, A_{2}\right)}{\left|A_{1}\right|-\left|A_{2}\right|}<$ alpha. For this learning algorithm, alpha is the unique parameter; we tuned it to get better quality automata.

\subsection{Optimizing Automata}

We use three measures to evaluate the quality of a probabilistic automaton (and set the value of alpha optimally). The first, called test sample perplexity (PP), is based on the per symbol loglikelihood of strings $x$ belonging to a test sample according to the distribution defined by the automaton. Formally, $L L=-\frac{1}{|S|} \sum_{x \in S} \log (P(x))$, where $P(x)$ is the probability assigned to the string $x$ by the automata. The perplexity PP is defined as $P P=2^{L L}$. The minimal perplexity $P P=1$ is reached when the next symbol is always predicted with probability 1 from the current state, while $P P=|\Sigma|$ corresponds to uniformly guessing from an alphabet of size $|\Sigma|$.

The second measure we used to evaluate the quality of an automaton is the number of missed samples (MS). A missed sample is a string in the test sample that the automaton failed to accept. One such instance suffices to have PP undefined (LL infinite). Since an undefined value of PP only witnesses the presence of at least one MS we decided to count the number of MS separately, and compute PP without taking MS into account. This choice leads to a more accurate value of PP, while, moreover, the value of MS provides us with information about the generalization capacity of automata: the lower the value of MS, the larger the generalization capacities of the automaton. The usual way to circumvent undefined perplexity is to smooth the resulting automaton with unigrams, thus increasing the generalization capacity of the automaton, which is usually paid for with an increase in perplexity. We decided not to use any smoothing techniques as we want to compare bigram-based automata with MDI-based automata in the cleanest possible way. The PP and MS measures are relative to a test sample; we transformed section 00 of the PTB to obtain one. ${ }^{1}$

\footnotetext{
${ }^{1}$ If smoothing techniques are used for optimizing automata based on $n$-grams, they should also be used for optimizing MDI-based automata. A fair experiment for comparing the two automata-learning algorithms using smoothing techniques would consist of first building two pairs of automata. The first pair would consist of the unigram-based automaton together
} 
The third measure we used to evaluate the quality of automata concerns the size of the automata. We compute NumEdges and NumStates (the number of edges and the number of states of the automaton).

We used PP, US, NumEdges, and NumStates to compare automata. We say that one automaton is of a better quality than another if the values of the 4 indicators are lower for the first than for the second. Our aim is to find a value of alpha that produces an automaton of better quality than the bigram-based counterpart. By exhaustive search, using all training data, we determined the optimal value of alpha. We selected the value of alpha for which the MDI-based automaton outperforms the bigram-based one. ${ }^{2}$

We exemplify our procedure by considering automata for the "One-Automaton" setting (where we used the same automata for all parts of speech). In Figure 3 we plot all values of PP and MS computed for different values of alpha, for each training set (i.e., left and right). From the plots we can identify values of a lpha that produce automata having better values of PP and MS than the bigram-based ones. All such alphas are the ones inside the marked areas; automata induced using those alphas possess a lower value of PP as well as a smaller number of MS, as required. Based on these explorations

\begin{tabular}{c||cc|cc}
\multicolumn{1}{c||}{} & \multicolumn{2}{c|}{ MDI } & \multicolumn{2}{c}{ Bigrams } \\
\hline & Right & Left & Right & Left \\
\hline NumEdges & 268 & 328 & 20519 & 16473 \\
NumStates & 12 & 15 & 844 & 755
\end{tabular}

Table 1: Automata sizes for the "One-Automaton" case, with alpha $=0.0001$.

we selected alpha $=0.0001$ for building the automata used for grammar induction in the "OneAutomaton" case. Besides having lower values of PP and MS, the resulting automata are smaller than the bigram based automata (Table 1). MDI compresses information better; the values in the tables

with an MDI-based automaton outperforming the unigrambased one. The second one, a bigram-based automata together with an MDI-based automata outperforming the bigram-based one. Second, the two $n$-gram based automata smoothed into a single automaton have to be compared against the two MDIbased automata smoothed into a single automaton. It would be hard to determine whether the differences between the final automata are due to smoothing procedure or to the algorithms used for creating the initial automata. By leaving smoothing out of the picture, we obtain a clearer understanding of the differences between the two automata induction algorithms.

${ }^{2}$ An equivalent value of alpha can be obtained independently of the performance of the bigram-based automata by defining a measure that combines PP and MS. This measure should reach its maximum when PP and MS reach their minimums. suggest that MDI finds more regularities in the sample set than the bigram-based algorithm.

To determine optimal values for the "ManyAutomata" case (where we learned two automata for each POS) we used the same procedure as for the "One-Automaton" case, but now for every individual POS. Because of space constraints we are not able to reproduce analogues of Figure 3 and Table 1 for all parts of speech. Figure 4 contains representative plots; the remaining plots are available online at http://www.science. uva.nl/ infante/Pos.

Besides allowing us to find the optimal alphas, the plots provide us with a great deal of information. For instance, there are two remarkable things in the plots for VBP (Figure 4, second row). First, it is one of the few examples where the bigrambased algorithm performs better than the MDI algorithm. Second, the values of PP in this plot are relatively high and unstable compared to other POS plots. Lower perplexity usually implies better quality automata, and as we will see in the next section, better automata produce better parsers. How can we obtain lower PP values for the VBP automata? The class of words tagged with VBP harbors many different behaviors, which is not surprising, given that verbs can differ widely in terms of, e.g., their subcategorization frames. One way to decrease the PP values is to split the class of words tagged with VBP into multiple, more homogeneous classes. Note from Figures 3 and 4 that splitting the original sample sets into POS-dependent sets produces a huge decrease on PP. One attempt to implement this idea is lexicalization: increasing the information in the POS tag by adding the lemma to it (Collins, 1997; Sima'an, 2000). Lexicalization splits the class of verbs into a family of singletons producing more homogeneous classes, as desired. A different approach (Klein and Manning, 2003) consists in adding head information to dependents; words tagged with VBP are then split into classes according to the words that dominate them in the training corpus.

Some POS present very high perplexities, but tags such as DT present a PP close to 1 (and $0 \mathrm{MS}$ ) for all values of alpha. Hence, there is no need to introduce further distinctions in DT, doing so will not increase the quality of the automata but will increase their number; splitting techniques are bound to add noise to the resulting grammars. The plots also indicate that the bigram-based algorithm captures them as well as the MDI algorithm.

In Figure 4, third row, we see that the MDI-based automata and the bigram-based automata achieve the same value of PP (close to 5) for NN, but 
Unique Automaton - Left Side

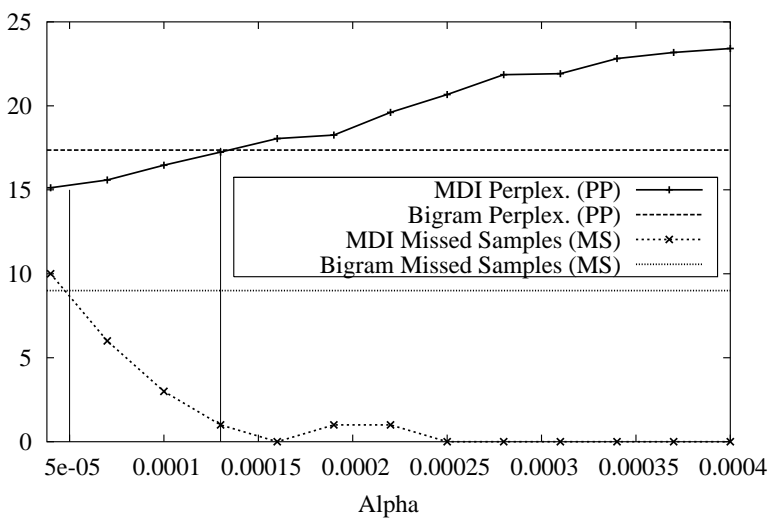

Unique Automaton - Right Side

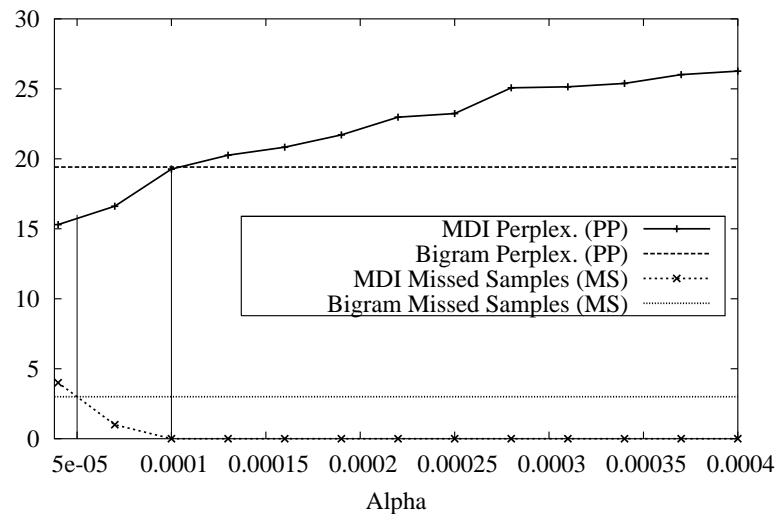

Figure 3: Values of PP and MS for automata used in building One-Automaton grammars. (X-axis): a lpha. (Y-axis): missed samples (MS) and perplexity (PP). The two constant lines represent the values of PP and MS for the bigram-based automata.
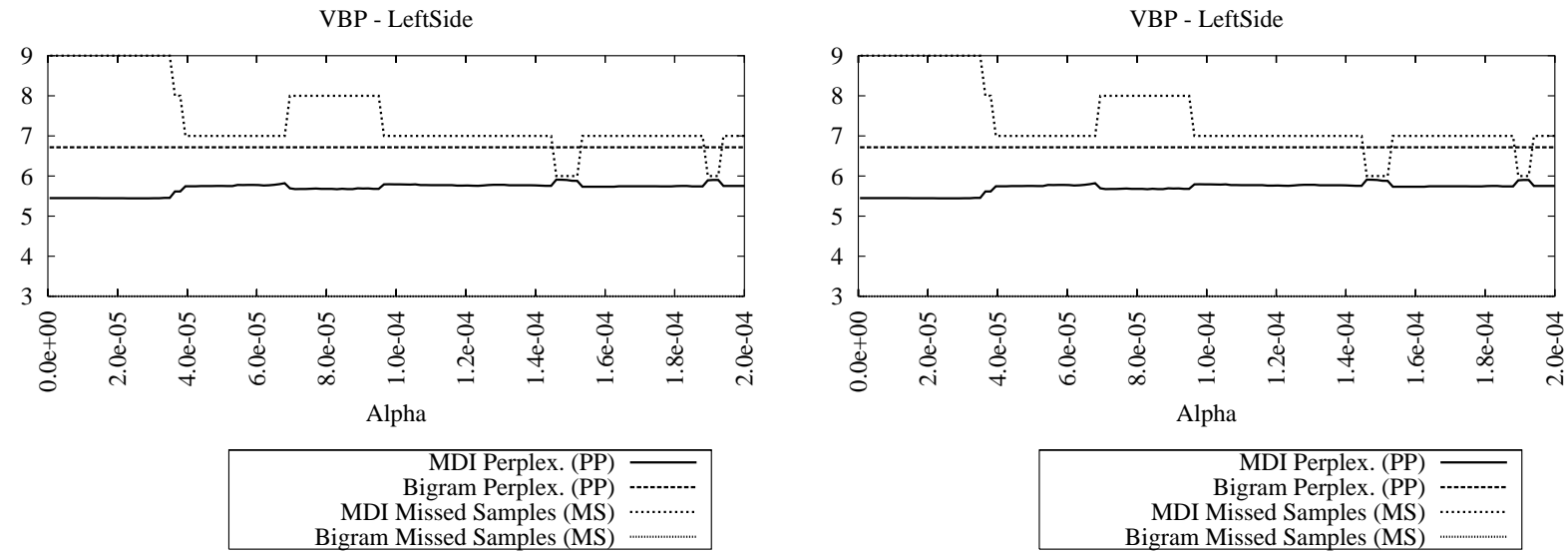

NN - LeftSide
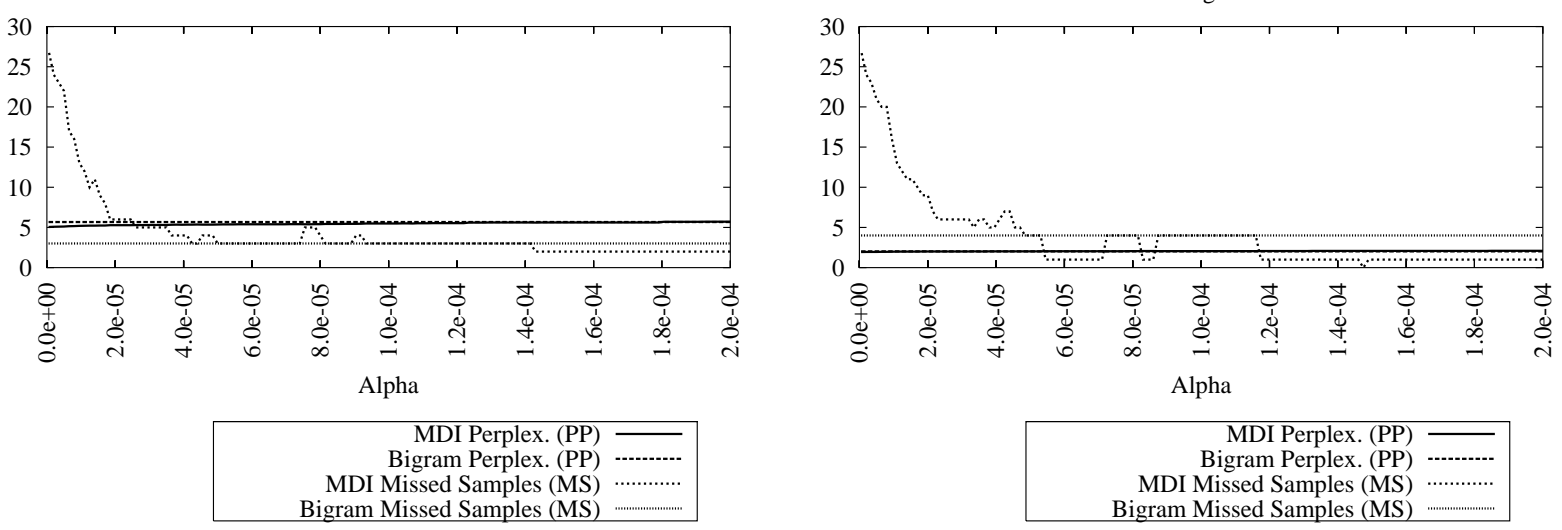

Figure 4: Values of PP and MS for automata for ad-hoc automata

the MDI misses fewer examples for alphas bigger than $1.4 e-04$. As pointed out, we built the One-Automaton-MDI using alpha $=0.0001$ and even though the method allows us to fine-tune each alpha in the Many-Automata-MDI grammar, we used a fixed alpha $=0.0002$ for all parts of speech, which, for most parts of speech, produces better au- tomata than bigrams. Table 2 lists the sizes of the automata. The differences between MDI-based and bigram-based automata are not as dramatic as in the "One-Automaton" case (Table 1), but the former again have consistently lower NumEdges and NumStates values, for all parts of speech, even where bigram-based automata have a lower perplexity. 


\begin{tabular}{cl||cc|cc}
\multicolumn{1}{l||}{} & \multicolumn{2}{c|}{ MDI } & \multicolumn{2}{c}{ Bigrams } \\
\cline { 3 - 6 } POS & & Right & Left & Right & Left \\
\hline DT & NumEdges & 21 & 14 & 35 & 39 \\
& NumStates & 4 & 3 & 25 & 17 \\
\hline VBP & NumEdges & 300 & 204 & 2596 & 1311 \\
& NumStates & 50 & 45 & 250 & 149 \\
\hline NN & NumEdges & 104 & 111 & 3827 & 4709 \\
& NumStates & 6 & 4 & 284 & 326
\end{tabular}

Table 2: Automata sizes for the three parts of speech in the "Many-Automata" case, with alpha = 0.0002 for parts of speech.

\section{Parsing the PTB}

We have observed remarkable differences in quality between MDI-based and bigram-based automata. Next, we present the parsing scores, and discuss the meaning of the measures observed for automata in the context of the grammars they produce. The measure that translates directly from automata to grammars is automaton size. Since each automaton is transformed into a PCFG, the number of rules in the resulting grammar is proportional to the number of arcs in the automaton, and the number of nonterminals is proportional to the number of states. From Table 3 we see that MDI compresses information better: the sizes of the grammars produced by the MDI-based automata are an order of magnitude smaller that those produced using bigram-based automata. Moreover, the "One-Automaton" versions substantially reduce the size of the resulting grammars; this is obviously due to the fact that all POS share the same underlying automaton so that information does not need to be duplicated across parts of speech. To understand the meaning of PP and

\begin{tabular}{cc|cc}
\multicolumn{2}{c|}{ One Automaton } & \multicolumn{2}{c}{ Many Automata } \\
\hline MDI & Bigram & MDI & Bigram \\
\hline 702 & 38670 & 5316 & 68394 \\
\hline
\end{tabular}

Table 3: Number of rules in the grammars built.

MS in the context of grammars it helps to think of PCW-parsing as a two-phase procedure. The first phase consists of creating the rules that will be used in the second phase. And the second phase consists in using the rules created in the first phase as a PCFG and parsing the sentence using a PCF parser. Since regular expressions are used to build rules, the values of PP and MS quantify the quality of the set of rules built for the second phase: MS gives us a measure of the number rule bodies that should be created but that will not be created, and, hence, it gives us a measure of the number of "correct" trees that will not be produced. PP tells us how uncertain the first phase is about producing rules.
Finally, we report on the parsing accuracy. We use two measures, the first one (\%Words) was proposed by Lin (1995) and was the one reported in (Eisner, 1996). Lin's measure computes the fraction of words that have been attached to the right word. The second one (\%POS) marks as correct a word attachment if, and only if, the POS tag of the head is the same as that of the right head, i.e., the word was attached to the correct word-class, even though the word is not the correct one in the sentence. Clearly, the second measure is always higher than the first one. The two measures try to capture the performance of the PCW-parser in the two phases described above: (\%POS) tries to capture the performance in the first phase, and (\%Words) in the second phase. The measures reported in Table 4 are the mean values of (\%POS) and (\%Words) computed over all sentences in section 23 having length at most 20 . We parsed only those sentences because the resulting grammars for bigrams are too big: parsing all sentences without any serious pruning techniques was simply not feasible. From Table 4

\begin{tabular}{c||cc|cc}
\multicolumn{1}{c||}{} & \multicolumn{2}{c|}{ MDI } & \multicolumn{2}{c}{ Bigrams } \\
\hline & \%Words & \%POS & \%Words & \%POS \\
\hline One-Aut. & 0.69 & 0.73 & 0.59 & 0.63 \\
Many-Aut. & 0.85 & 0.88 & 0.73 & 0.76
\end{tabular}

Table 4: Parsing results for the PTB

we see that the grammars induced with MDI outperform the grammars created with bigrams. Moreover, the grammar using different automata per POS outperforms the ones built using only a single automaton per side (left or right). The results suggest that an increase in quality of the automata has a direct impact on the parsing performance.

\section{Related Work and Discussion}

Modeling rule bodies is a key component of parsers. $N$-grams have been used extensively for this purpose (Collins 1996, 1997; Eisner, 1996). In these formalisms the generative process is not considered in terms of probabilistic regular languages. Considering them as such (like we do) has two advantages. First, a vast area of research for inducing regular languages (Carrasco and Oncina, 1994; Thollard et al., 2000; Dupont and Chase, 1998) comes in sight. Second, the parsing device itself can be viewed under a unifying grammatical paradigm like PCW-grammars (Chastellier and Colmerauer, 1969; Infante-Lopez and de Rijke, 2003). As PCWgrammars are $\mathrm{PCFGs}$ plus post tree transformations, properties of PCFGs hold for them too (Booth and Thompson, 1973). 
In our comparison we optimized the value of alpha, but we did not optimize the $n$-grams, as doing so would mean two different things. First, smoothing techniques would have to be used to combine different order $n$-grams. To be fair, we would also have to smooth different MDI-based automata, which would leave us in the same point. Second, the degree of the $n$-gram. We opted for $n=2$ as it seems the right balance of informativeness and generalization. $N$-grams are used to model sequences of arguments, and these hardly ever have length $>3$, making higher degrees useless. To make a fair comparison for the Many-Automata grammars we did not tune the MDI-based automata individually, but we picked a unique alpha.

MDI presents a way to compact rule information on the PTB; of course, other approaches exists. In particular, Krotov et al. (1998) try to induce a $\mathrm{CW}$-grammar from the PTB with the underlying assumption that some derivations that were supposed to be hidden were left visible. The attempt to use algorithms other than $n$-grams-based for inducing of regular languages in the context of grammar induction is not new; for example, Kruijff (2003) uses profile hidden models in an attempt to quantify free order variations across languages; we are not aware of evaluations of his grammars as parsing devices.

\section{Conclusions and Future Work}

Our experiments support two kinds of conclusions. First, modeling rules with algorithms other than $n$-grams not only produces smaller grammars but also better performing ones. Second, the procedure used for optimizing alpha reveals that some POS behave almost deterministically for selecting their arguments, while others do not. These findings suggests that splitting classes that behave nondeterministically into homogeneous ones could improve the quality of the inferred automata. We saw that lexicalization and head-annotation seem to attack this problem. Obvious questions for future work arise: Are these two techniques the best way to split non-homogeneous classes into homogeneous ones? Is there an optimal splitting?

\section{Acknowledgments}

We thank our referees for valuable comments. Both authors were supported by the Netherlands Organization for Scientific Research (NWO) under project number 220-80-001. De Rijke was also supported by grants from NWO, under project numbers 365 20-005, 612.069.006, 612.000.106, 612.000.207, and 612.066.302.

\section{References}

S. Abney, D. McAllester, and F. Pereira. 1999. Relating probabilistic grammars and automata. In Proc. 37th Annual Meeting of the ACL, pages 542-549.

T. Booth and R. Thompson. 1973. Applying probability measures to abstract languages. IEEE Transaction on Computers, C-33(5):442-450.

R. Carrasco and J. Oncina. 1994. Learning stochastic regular grammars by means of state merging method. In Proc. ICGI-94, Springer, pages 139-150.

E. Charniak. 1997. Statistical parsing with a contextfree grammar and word statistics. In Proc. 14th Nat. Conf. on Artificial Intelligence, pages 598-603.

G. Chastellier and A. Colmerauer. 1969. W-grammar. In Proc. 1969 24th National Conf., pages 511-518.

M. Collins. 1996. A new statistical parser based on bigram lexical dependencies. In Proc. 34th Annual Meeting of the ACL, pages 184-191.

M. Collins. 1997. Three generative, lexicalized models for statistical parsing. In Proc. 35th Annual Meeting of the ACL and 8th Conf. of the EACL, pages 16-23.

M. Collins. 1999. Head-Driven Statistical Models for Natural Language Parsing. Ph.D. thesis, University of Pennsylvania, PA.

M. Collins. 2000. Discriminative reranking for natural language parsing. In Proc. ICML-2000, Stanford, Ca.

T. Cover and J. Thomas. 1991. Elements of Information Theory. Jonh Wiley and Sons, New York.

F. Denis. 2001. Learning regular languages from simple positive examples. Machine Learning, 44(1/2):37-66.

P. Dupont and L. Chase. 1998. Using symbol clustering to improve probabilistic automaton inference. In Proc. ICGI-98, pages 232-243.

J. Eisner. 1996. Three new probabilistic models for dependency parsing: An exploration. In Proc. COLING96, pages 340-245, Copenhagen, Denmark.

J. Eisner. 2000. Bilexical grammars and their cubic-time parsing algorithms. In Advances in Probabilistic and Other Parsing Technologies, pages 29-62. Kluwer.

E. M. Gold. 1967. Language identification in the limit. Information and Control, 10:447-474.

G. Infante-Lopez and M. de Rijke. 2003. Natural language parsing with $\mathrm{W}$-grammars. In Proc. CLIN 2003.

D. Klein and C. Manning. 2003. Accurate unlexicalized parsing. In Proc. 41st Annual Meeting of the ACL.

A. Krotov, M. Hepple, R.J. Gaizauskas, and Y. Wilks. 1998. Compacting the Penn Treebank grammar. In Proc. COLING-ACL, pages 699-703.

G. Kruijff. 2003. 3-phase grammar learning. In Proc. Workshop on Ideas and Strategies for Multilingual Grammar Development.

D. Lin. 1995. A dependency-based method for evaluating broad-coverage parsers. In Proc. IJCAI-95.

K. Sima'an. 2000. Tree-gram Parsing: Lexical Dependencies and Structual Relations. In Proc. 38th Annual Meeting of the ACL, pages 53-60, Hong Kong, China.

F. Thollard, P. Dupont, and C. de la Higuera. 2000. Probabilistic DFA inference using kullback-leibler divergence and minimality. In Proc. ICML 2000. 